\title{
Artificial biomolecular condensates
}

\author{
Synthetic small molecules can form liquid-phase condensates and \\ simultaneously sequester intracellular enzymes.
}

$t$ is now well known that in addition to canonical membrane-bound organelles, eukaryotic cells also contain diverse membraneless compartments that enable spatial organization of molecular components for biochemical reactions. These membraneless compartments, such as ribonucleoprotein particle granules, have been shown to coalesce into droplets with liquid-like features surrounded by dynamic intracellular environments.

More evidence suggests that these liquid condensates are ubiquitous and have a critical role in cell functions. For example, Boija et al. (2018) show that activation domains from transcription factors can form phase-separated condensates with the Mediator coactivator for gene activation. Yet it is still largely unknown how cells organize their biomolecules spatially and dynamically to precisely control cellular functions.

These membraneless compartments are challenging to study because of the complicated intracellular environment, which makes it difficult to dissect the molecular interactions that drive phase transition. Researchers are tackling the problem from all angles in a variety of ways, from the application of polymer physics to understand the molecular interactions, to the development of an optogenetic system to manipulate the condensate process, to the synthesis of molecules that self-assemble into intracellular condensates.

Among the approaches, cell-free assays offer a straightforward way to control the composition of a given molecular condensate and thus help to reveal the roles of molecular interactions in phase transition. To this end, polypeptides with unique chemical compositions have been recombinantly synthesized to probe the effects of electrostatic, dipolar, and hydrophobic interaction on the formation of biocondensates.

Bing $\mathrm{Xu}$ and his team from Brandeis University have a long-standing interest in intracellular soluble-to-gel (sol-to-gel) transition. In 2007, they explored the formation of intracellular hydrogelation from small molecules and its effects on cell functions. "We have shown the liquid-phase separation of small peptides can selectively cause apoptosis of cancer cells," notes Xu.
Now they utilize this sol-to-gel transition to mimic the liquid-phase condensates in cells (Feng et al., 2018). Instead of overexpressing recombinant polypeptides, $\mathrm{Xu}$ and his team used solid-phase synthesis to generate modified short peptides that can act as a precursor for instructed self-assemblies. The precursor consists of a self-assembling peptide backbone, as well as a drug and a ligand to trigger the dephosphorylating enzymatic reaction that converts the precursor into the hydrogelator. The precursor and hydrogelator subsequently coassemble to promote the association with cyclooxygenase-2 (COX-2) and protein tyrosine phosphatase $1 \mathrm{~B}$ (PTP1B) on endoplasmic reticulum.

As a proof of principle, they examined the sol-to-gel transition in vitro. The addition of alkaline phosphatase triggers the formation of hydrogelator, forming short nanofibers that later coassemble with the precursor into long, uniform nanofibers. To demonstrate intracellular biocondensate formation, they incubated Saos- 2 cells with the precursor, and observed that both COX-2 and PTP1B formed puncta at the site of endoplasmic reticulum. This synthetic approach thus allows local concentration of enzymes of interest at specific sites.

The ability to synthesize molecules with distinct chemical compositions allows researchers to manipulate the molecular interactions and tune the macroscopic properties of the condensates. The generation of intracellular condensates from such molecules offers an alternative approach to elucidate the molecular mechanism underlying cellular regulation of liquid-liquid phase separation. "Designing and using the synthetic molecules helps us better understand how the spatiotemporal behaviors of liquid phase separation control cellular functions," says Xu.

\section{Lei Tang}

Published online: 20 December 2018 https://doi.org/10.1038/s41592-018-0288-4

\section{Research papers}

Boija, A. et al. Transcription factors activate genes through the phase-separation capacity of their activation domains. Cell https://doi.org/10.1016/j. cell.2018.10.042 (2018)

Feng, Z., Wang, H. \& Xu, B. Instructed assembly of peptides for intracellular enzyme sequestration. J. Am. Chem. Soc. 140, 16433-16437 (2018). natureresearch
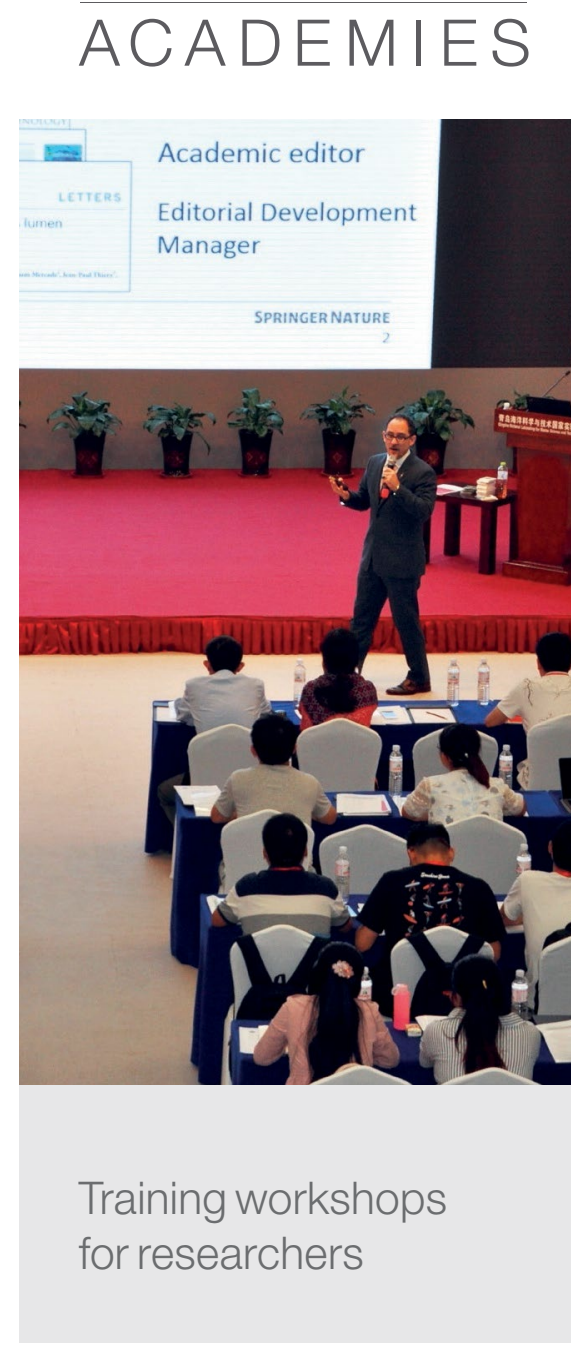

\author{
A series of workshops \\ to support researchers, \\ covering topics such as \\ getting published, journal \\ editing, clinical research \\ methodology, and \\ applying for research \\ positions.
}

\author{
Visit \\ partnerships.nature. \\ com/academies to host \\ an academy at your \\ institution.
}

